\title{
Wide local excision and reconstructive surgery of desmoid tumour of the crus (Case report)
}

\author{
CHENG CAO* , JUN ZOU* ${ }^{*}$, CHUNSHEN WU, ZHAOHUA BAO and HUILIN YANG \\ Department of Orthopaedic Surgery, The First Affiliated Hospital of Soochow University, Suzhou, Jiangsu 215006, P.R. China
}

Received February 22, 2012; Accepted May 21, 2012

DOI: $10.3892 / \mathrm{ol} .2012 .766$

\begin{abstract}
Extra-abdominal desmoid tumour is a rare type of tumour which is conventionally treated with wide local excision. However, wide local excision usually causes irreparable damage to the limbs. Nevertheless, few patients have been subjected to wide local excision and reconstructive surgery in a single procedure. A 52-year-old female reported a slow-growing lump in the right crus which had been growing for two years. The patient complained of persistent pain, especially at night, and was first subjected to lump resection in another hospital. The postoperative histopathological examination indicated muscle fibroma. Three months later, the lump recurred in the same position. The persistent pain induced by the tumour hampered her ability to walk. Wide local excision was conducted on the major parts of the gastrocnemius and soleus. After the pathological examination confirmed that the resection margin was negative, we performed reconstructive surgery on the Achilles tendon. The patient recovered plantarflexion function following the surgery and did not report any recurrence in the 6-year follow-up period. The desmoid tumour is a low-grade malignant tumour. Thus, the main focus of the treatment is to restore the function of the limbs to optimal capacity, such that the incidence of tumour recurrence is minimised.
\end{abstract}

\section{Introduction}

Desmoid tumour, also known as desmoid fibromatosis, is a rare (3-4 cases/million individuals/year), deep-seated, musculoaponeurotic tumour which accounts for $0.03 \%$ of all neoplasms (1-2). Females are more susceptible to developing this tumour than males, and the tumour may occur at any

Correspondence to: Professor Huilin Yang, Department of Orthopaedic Surgery, The First Affiliated Hospital of Soochow University, 188 Shizi St., Suzhou, Jiangsu 215006, P.R. China

E-mail: hlyang@suda.edu.cn

*Contributed equally

Key words: desmoid tumour, surgery, aggressive fibromitosis, wide resection age (3). The tumour usually develops in the following three areas: i) extra-abdominal area of the shoulder and pelvic girdle or chest and neck wall; ii) abdominal area of wall muscles; iii) intra-abdominal area of the pelvis, mesentery connective tissue and retroperitoneal space (4).

The natural history of desmoid tumours remains unknown. The tumours usually grow slowly, but in certain cases they may enlarge rapidly. Some of these tumours may stop growing and regress naturally $(5,6)$. For this reason, desmoid tumours have been referred to as enigmatic. The etiology of this disease is not well-defined: trauma, genetic disposition and oestrogen imbalance are considered to be factors responsible for this disease. Wide local excision is the preferred method of treatment, but it often causes serious damage to the limb function. In this study, we investigate a rare case, wherein wide local excision and reconstructive surgery were conducted in a single procedure.

The study was approved by the ethics committee of the First Affiliated Hospital of Soochow University, China.

\section{Case report}

A 52-year-old female reported a slow-growing lump in the right crus, which had been increasing in size for two years. The patient complained of persistent pain, especially at night. The patient was subjected to lump resection in another hospital, wherein the postoperative histopathological examination showed muscle fibroma. Following surgery, the patient demonstrated signs of excellent recovery. Three months later, the lump recurred in the same position. The persistent pain associated with the lump impeded her ability to walk, and she did not report any other complaints. Examination revealed an irregular tumour $10 \times 8 \mathrm{~cm}$ in size with tough texture, poor mobility, blurred border and moderate tenderness. The results of other tests were negative.

The MRI scan revealed a homogeneously iso-signal intensity lesion on the T1-weighted image and a heterogeneous high intensity signal was detected on the T2-weighted image. The contrast-enhanced T1-weighted axial images showed mild enhancement of the lesion through an irregular margin. The MRI scan also revealed bands of low signal intensity within the lesion. In addition, desmoid tumours did not reveal any central necrosis, even in the largest lesions (Fig. 1).

Wide local excision through excision biopsy of the tumour was planned. Under the spinal anaesthesia effect, with the 


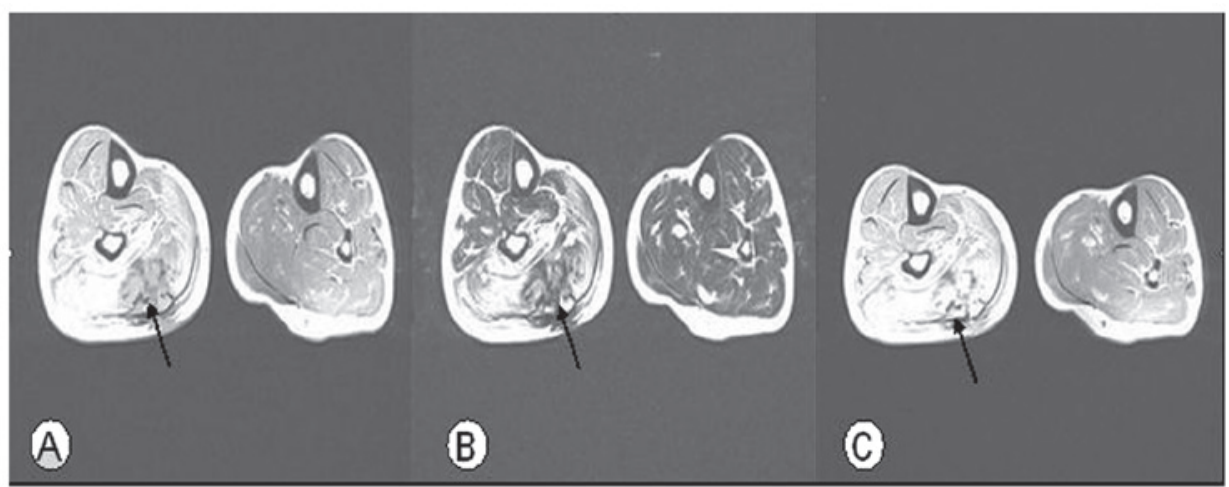

Figure 1. Magnetic resonance imaging (MRI) of a 52-year-old female with desmoid tumour in the right crus. (A) T1-weighted axial image shows homogeneous iso-signal intensity. (B) T2-weighted axial image shows heterogeneously high signal intensity. (C) The contrast-enhanced T1-weighted axial image shows mild enhancement of the lesion with an irregular margin, and the low signal intensity on all pulse sequences within the lesion (arrow). In addition, the lesion did not show central necrosis.

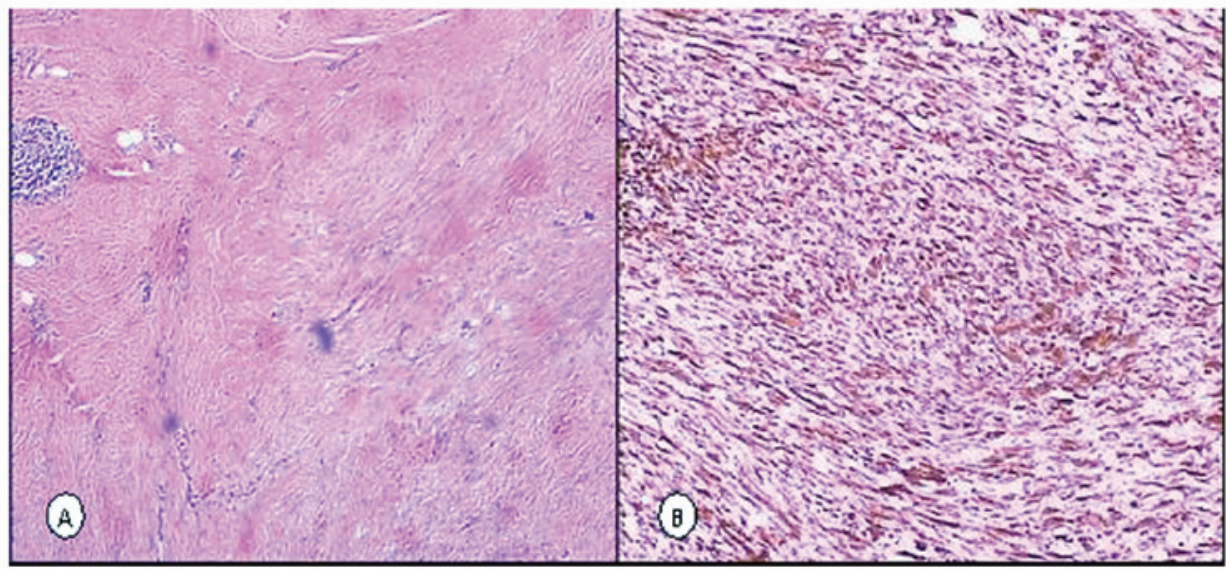

Figure 2. Histopathological findings of a soleus-based and gastrocnemius-based desmoid tumour resected from a 52-year-old woman. (A) Hematoxylin and eosin staining showing (right crus) ligament sample fibroma. (B) Histiocytes showing positive staining for Vimentin.

patient in a prone position, an arc incision of approximately $20 \mathrm{~cm}$ was made along the long axis of the back side through the right crus. The tumour was attached to the soleus and had infiltrated into most of the gastrocnemius. The size of the tumour was approximately $8 \times 8 \mathrm{~cm}$. The tumour was firm; however, it had an irregular margin and lacked a capsule. We exposed the posterior tibial nerve and posterior tibial blood vessel, which were not affected by the tumour. We removed the medial and lateralis heads of the gastrocnemius from the fringe. The major part of the soleus muscle belly was resected. Then, the junction of the Achilles tendon, gastrocnemius and soleus was removed. A small part of the lateralis was left. The upper and lower ends of the residual muscles were subjected to fast pathological examination. The fast pathological examination confirmed that the resection margin was negative. We made a longitudinal incision in the Achilles tendon, wherein half of the Achilles tendon was included as an extension to rebuild the Achilles tendon. Postoperatively, a cast immobilisation was undertaken in the plantarflexion position for approximately 4 weeks. Postoperative histopathological examination of the right crus revealed ligament sample fibroma. Immunohistochemical examination revealed that staining for Vimentin was positive, while that for CD34 and SMA were negative (Fig. 2). Following the surgery, the

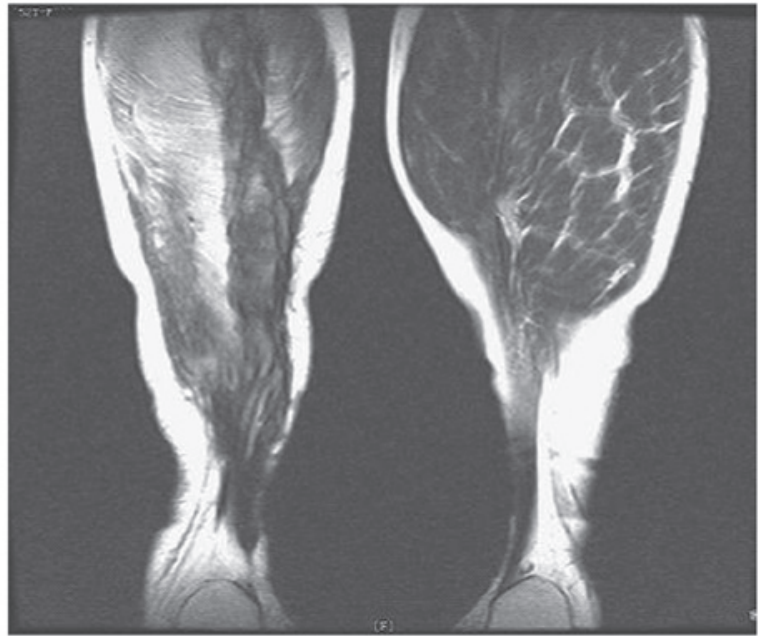

Figure 3. Magnetic resonance image 4 months after resection of the tumour and reconstructive surgery of the Achilles tendon. The image indicates the absence of recurrence.

symptoms improved markedly and the patient partially recovered plantarflexion function. After 4 months, the follow-up MRI examination did not reveal any recurrence of the tumour 
(Fig. 3). In the follow-up period of six years, the patient did not show any sign of recurrence.

\section{Discussion}

Extra-abdominal desmoid tumour is a fibroblastic tumour which arises from the connective tissues of muscles, fascia, aponeurosis or periosteum (7). Despite their benign appearance, these are locally aggressive tumours, which tend to invade the surrounding structures as they lack a true capsule. Therefore, these tumours are often misclassified as low-grade malignancies (low-grade malignant tumours) (8). The tumour is not known to metastasise, but it has a high recurrence rate after surgery. A local recurrence rate of $24-77 \%$ has been reported at 10 years.

Current treatments include radiation therapy, chemotherapy, drug therapy and hormone therapy. However, surgical resection is the preferred method of treatment. As the disease has the clinical characteristics of an aggressive growth and a high recurrence rate, the tumour should be widely resected. Wide local excision with a negative margin is considered to be a significant factor that decreases the recurrence rate. Ballo et al (9) performed a retrospective review of 189 consecutive cases of desmoid tumour that were treated only with surgical resection. Among the patients with negative margins, the 10 -year recurrence rate was $27 \%$. However, 40 patients with positive margins had a 10 -year relapse rate of $54 \%$. Leithner et al (10) performed a comparative analysis based on the data extrapolated from 412 retrospective studies: 152 patients with negative margins had a recurrence rate of $27 \%$, while 260 patients with positive margins had a local recurrence rate of $72 \%$. Therefore, the quality of the resection boundary is considered to be the most significant factor for predicting the recurrence of the tumour. If resection achieves a negative margin, the postoperative recurrence rate is relatively low. However, certain groups have reported that the recurrence rate remains high following wide local excision with a negative margin. We suggest two reasons for this observation. Firstly, desmoid tumours lack a pseudocapsule and form a nonpalpable mass that infiltrates along the muscle bundles and fascia. Moreover, it is difficult to detect the exact localisation and extension of infiltration or dissemination of the lesion in the tumour. Secondly, the negative margin is observed with the naked eye instead of by pathological examination, which may not reflect the true margin. In this case, we examined the upper and lower ends of residual muscles for fast pathological examination. The pathological examination confirmed that the resection margin was negative.

However, wide local excision causes serious damage to limb function. However, selecting the appropriate treatment method remains a challenge. Ferraresi et al (11) described a case wherein the tumour had selectively invaded the patient's radial nerve. The tumour was partially removed with the radial nerve and neurotransplantation was performed. This procedure showed an excellent clinical recovery and no recurrence was reported in the 6-year follow-up period. Gallucci et al (12) presented a case of aggressive fibromatosis in the proximal third of the forearm that was treated with wide resection and reconstructive surgery in a single procedure. An acceptable functional result did not show any evidence of recurrence within the 3-year follow-up period. Thus, wide local excision should be undertaken to reduce the recurrence rate of tumours. Reconstructive surgery proves to be of little significance if the tumour relapses.

In this case, the tumour had a wide range. We resected most of the gastrocnemius and soleus. After performing a pathological examination, we conducted reconstructive surgery of the Achilles tendon. We suggest that reconstructive surgery should be performed to maintain the functioning of the lower limb. The patient recovered part of the plantarflexion function after the surgery and had no recurrence in the 6-year follow-up period.

In conclusion, we consider that wide local excision with a negative margin by pathological examination reduces the recurrence rate. As desmoid tumours are low-grade malignant tumours, we need to maintain the optimum function of limbs as far as possible. This reduces the recurrence rate of the tumours.

\section{References}

1. Berri RN, Baumann DP, Madewell JE, et al: Desmoid tumor: current multidisciplinary approaches. Ann Plast Surg 67: 551-564, 2011.

2. Shields CJ, Winter DC, Kirwan WO and Redmond HP: Desmoid tumor. Eur J Surg Oncol 27: 701-706, 2001.

3. Meazza C, Bisogno G, Gronchi A, et al: Aggressive fibromatosis in children and adolescents: the Italian experience. Cancer 116: 233-240, 2010.

4. Ferenc T, Sygut J, Kopczyński J, et al: Aggressive fibromatosis (desmoid tumors): definition, occurrence, pathology, diagnostic problems, clinical behavior, genetic background. Pol J Pathol 57: 5-15, 2006.

5. Okuno S: The enigma of desmoid tumors. Curr Treat Options Oncol 7: 438-443, 2006.

6. Dalén BP, Geijer M, Kvist H, et al: Clinical and imaging observations of desmoid tumors left without treatment. Acta Orthop 77: 932-937, 2006.

7. Agrawal PS, Jagtap SM and Mitra SR: Extra-abdominal desmoid tumour of the leg. Singapore Med J 49: e6-e7, 2008.

8. Merchant NB, Lewis JJ, Woodruff JM, et al: Extremity and trunk desmoid tumors: a multifactorial analysis of outcome. Cancer 86: 2045-2052, 1999.

9. Ballo MT, Zagars GK, Pollack A, et al: Desmoid tumor: prognostic factors and outcome after surgery, radiation therapy, or combined surgery and radiation therapy. J Clin Oncol 17: 158-167, 1999.

10. Leithner A, Gapp M, Leithner K, et al: Margins in extraabdominal desmoid tumors: A comparative analysis. J Surg Oncol 86: $152-156,2004$

11. Ferraresi S, Garozzo D and Bianchini E: Aggressive fibromatosis (desmoid tumor) of the radial nerve: favorable resolution. Case report. J Neurosurg 95: 332-333, 2001.

12. Gallucci GL, Boretto JG and De Carli P: Desmoid tumor of the forearm. Reconstructive surgery and functional result. Chir Main 28: 326-329, 2009. 